PROCEEDINGS OF THE

AMERICAN MATHEMATICAL SOCIETY

Volume 134, Number 5, Pages 1271-1275

S 0002-9939(05)08174-8

Article electronically published on October 13, 2005

\title{
CHARACTERIZATION OF MODULES OF FINITE PROJECTIVE DIMENSION OVER COMPLETE INTERSECTIONS
}

\author{
JINJIA LI
}

(Communicated by Bernd Ulrich)

\begin{abstract}
Let $M$ be a finitely generated module over a local complete intersection $R$ of characteristic $p>0$. The property that $M$ has finite projective dimension can be characterized by the vanishing of $\operatorname{ext}_{R}^{i}\left(f^{n} R, M\right)$ for some $i>0$ and for some $n>0$.
\end{abstract}

Let $(R, m, k)$ be a local ring of characteristic $p>0$. The Frobenius endomorphism $f_{R}: R \rightarrow R$ is defined by $f_{R}(r)=r^{p}$ for $r \in R$. Each iteration $f_{R}^{n}$ defines a new $R$-module structure on $R$, denoted by $f^{n} R$, for which $a \cdot b=a^{p^{n}} b$. For any $R$-module $M, F_{R}^{n}(M)$ will stand for $M \otimes_{R} f^{n} R$ and $\widetilde{F}_{R}^{n}(M)$ will stand for $\operatorname{Hom}_{R}\left(f^{n} R, M\right)$. Avramov and Miller A-M proved that over a local complete intersection ring, if a finitely generated $R$-module $M$ satisfies $\operatorname{Tor}_{i}^{R}\left(M, f^{n} R\right)=0$ for some fixed $i, n>0$, then it is of finite projective dimension. Later, Dutta [D] provided a simple proof of this result without using the notion of "complexity". Using a similar method as in [D, we obtain another characterization of finitely generated modules of finite projective dimension over complete intersection rings.

Theorem. Let $M$ be a finitely generated module over a local complete intersection ring $R$. If for some $i, n>0$, $\operatorname{Ext}_{R}^{i}\left(f^{n} R, M\right)=0$, then $M$ has finite injective dimension over $R$.

Proof. Without loss of generality we can assume that $R$ is complete and the residue field $k$ is perfect. Let $R=S / \mathbf{x}$, where $S$ is a complete regular local ring of characteristic $p>0$ and $\mathbf{x}=\left(x_{1}, \ldots, x_{r}\right)$ is an ideal generated by an $S$-sequence $x_{1}, \ldots, x_{r}$. Write $R_{n}=S / \mathrm{x}^{p^{n}}$. Note that with this notation, every $R_{n}$-module is also an $R_{l}$-module via the natural surjection $R_{l} \rightarrow R_{n}$ for all $l>n$.

Let $\tilde{f}^{n}=f_{S}^{n} \otimes_{S} R$ (base change of $f_{S}^{n}: S \rightarrow S$ along the $S$-algebra $R$ ). We know by Kunz's Theorem [K] Theorem 3.3] that $f_{S}^{n}$ is flat. Since $k$ is perfect, $f_{S}^{n}$ is module finite. It follows that $\tilde{f}^{n}: S / \mathbf{x} \rightarrow S / \mathbf{x}^{p^{n}}$ is also flat and module finite. Observe that the map $R \stackrel{f_{R}^{n}}{\longrightarrow} R$ can be factored as

$$
S / \mathbf{x} \stackrel{\tilde{f}^{n}}{\longrightarrow} S / \mathbf{x}^{p^{n}} \stackrel{\eta_{n}}{\longrightarrow} S / \mathbf{x}
$$

Received by the editors October 4, 2004 and, in revised form, December 14, 2004.

2000 Mathematics Subject Classification. Primary 13C14, 13C40, 13D05, 13D40, 13 H10.

Key words and phrases. Complete intersection, finite projective dimension, finite injective dimension, flatness, Frobenius, Ext, Tor.

This research was carried out while the author was supported by a research grant from the UIUC Campus Research Board of the University of Illinois under the supervision of S. Dutta.

(C)2005 American Mathematical Society Reverts to public domain 28 years from publication 
or

$$
R \stackrel{\tilde{f}^{n}}{\longrightarrow} R_{n} \stackrel{\eta_{n}}{\longrightarrow} R
$$

where $\eta_{n}: S / \mathbf{x}^{p^{n}} \longrightarrow S / \mathbf{x}$ is the natural surjection. Thus $f_{R}^{n}=\eta_{n} \cdot \tilde{f}^{n}$.

It follows from the adjointness of Hom and Tensor that for any $R$-module $T$,

$$
\operatorname{Hom}_{R_{n}}\left(R, \operatorname{Hom}_{R}\left(\tilde{f}^{n} R_{n}, T\right)\right) \simeq \operatorname{Hom}_{R}\left(R \otimes_{R_{n}} \tilde{f}^{n} R_{n}, T\right) \simeq \operatorname{Hom}_{R}\left(f^{n} R, T\right)
$$

Let $I^{\bullet}$ be an injective resolution of $M$ over $R$. Since $\tilde{f}^{n}$ is flat, $\operatorname{Hom}_{R}\left(\widetilde{f}^{n} R_{n}, I^{\bullet}\right)$ is an injective resolution of $\operatorname{Hom}_{R}\left(\tilde{f}^{n} R_{n}, M\right)$ over $R_{n}$. Therefore, by (2)

$$
\begin{aligned}
\operatorname{Ext}_{R}^{j}\left(f^{n} R, M\right) & =H^{j}\left(\operatorname{Hom}_{R}\left(f^{n} R, I^{\bullet}\right)\right) \\
& \simeq H^{j}\left(\operatorname{Hom}_{R_{n}}\left(R, \operatorname{Hom}_{R}\left(\tilde{f}^{n} R_{n}, I^{\bullet}\right)\right)\right) \\
& =\operatorname{Ext}_{R_{n}}^{j}\left(R, \operatorname{Hom}_{R}\left(\tilde{f}^{n} R_{n}, M\right)\right), \forall j \geq 0,
\end{aligned}
$$

but

$$
\begin{aligned}
\operatorname{Hom}_{R}\left(\tilde{f}^{n} R_{n}, M\right) & =\operatorname{Hom}_{R}\left(f^{n} S \otimes_{s} R, M\right) \\
& \simeq \operatorname{Hom}_{S}\left(f^{n} S, M\right) \\
& =\widetilde{F}_{S}^{n}(M) .
\end{aligned}
$$

Hence, $\operatorname{Ext}_{R}^{j}\left(f^{n} R, M\right) \simeq \operatorname{Ext}_{R_{n}}^{j}\left(S / \mathbf{x}, \widetilde{F}_{S}^{n}(M)\right), \forall j \geq 0$.

Next, we show $\operatorname{Ext}_{R}^{i}\left(f^{n} R, M\right)=0$ for some $i>0$ implies $\operatorname{Ext}_{R}^{i}\left(f^{n} R, M\right)=0$ for all $i>0$. Consider a filtration of $S / \mathbf{x}^{p^{n}}$ of the form

$$
\begin{gathered}
0 \rightarrow K_{1} \rightarrow S / \mathbf{x}^{p^{n}} \rightarrow S / \mathbf{x} \rightarrow 0 \\
0 \rightarrow K_{2} \rightarrow K_{1} \rightarrow S / \mathbf{x} \rightarrow 0 \\
\vdots \\
0 \rightarrow K_{t_{n}} \rightarrow K_{t_{n-1}} \rightarrow S / \mathbf{x} \rightarrow 0
\end{gathered}
$$

where $K_{t_{n}}=S / \mathbf{x}$. By applying $\operatorname{Hom}_{R_{n}}\left(-, \widetilde{F}_{S}^{n}(M)\right)$ to the above short exact sequences, we get the long exact sequences of Ext's. Since $\operatorname{Ext}_{R_{n}}^{i}\left(S / \mathbf{x}, \widetilde{F}_{S}^{n}(M)\right)=0$, working from the last long exact sequence up to the first long exact sequence, we obtain that $\operatorname{Ext}_{R_{n}}^{i+1}\left(S / \mathbf{x}, \widetilde{F}_{S}^{n}(M)\right)=0$. Similarly, one can also obtain that $\operatorname{Ext}_{R_{n}}^{i-1}\left(S / \mathbf{x}, \widetilde{F}_{S}^{n}(M)\right)=0$ if $i>1$. Repeating these processes, we get

$$
\operatorname{Ext}_{R_{n}}^{i}\left(S / \mathbf{x}, \widetilde{F}_{S}^{n}(M)\right)=0
$$

for all $i>0$.

Then, we show $\operatorname{Ext}_{R}^{i}\left(f^{n} R, M\right)=0$ implies $\operatorname{Ext}_{R}^{i}\left(f^{n+1} R, M\right)=0, \forall i>0$. We need the following lemma.

Lemma. Let $A \rightarrow B$ be a ring homomorphism such that $B$ is a finitely generated free module over $A$. Let $M, N$ be A-modules. Then, as A-modules,

$$
\operatorname{Ext}_{B}^{i}\left(\operatorname{Hom}_{A}(B, M), \operatorname{Hom}_{A}(B, N)\right) \simeq \operatorname{Hom}_{A}\left(B, \operatorname{Ext}_{A}^{i}(M, N)\right) .
$$


Proof of the Lemma. Note that, as $A$-modules,

$$
\begin{aligned}
\operatorname{Hom}_{B} & \left(\operatorname{Hom}_{A}(B, M), \operatorname{Hom}_{A}(B, N)\right) \\
& \simeq \operatorname{Hom}_{A}\left(\operatorname{Hom}_{A}(B, M) \otimes_{B} B, N\right) \text { (adjointness) } \\
& \simeq \operatorname{Hom}_{A}\left(\operatorname{Hom}_{A}(B, M), N\right) \\
& \simeq \operatorname{Hom}_{A}\left(M \otimes_{A} B, N\right) \quad \text { (since } B \text { is } A \text {-free) } \\
& \simeq \operatorname{Hom}_{A}\left(B, \operatorname{Hom}_{A}(M, N)\right) .
\end{aligned}
$$

This proves the case when $i=0$. For $i>0$, let $J^{\bullet}$ be an injective resolution of $N$. Then $\operatorname{Hom}_{A}\left(B, J^{\bullet}\right)$ is an injective resolution of $\operatorname{Hom}_{A}(B, N)$ over $B$ since $B$ is free over $A$. So, as $A$-modules,

$$
\begin{aligned}
\operatorname{Ext}_{B}^{i} & \left(\operatorname{Hom}_{A}(B, M), \operatorname{Hom}_{A}(B, N)\right) \\
& =H^{i}\left(\operatorname{Hom}_{B}\left(\operatorname{Hom}_{A}(B, M), \operatorname{Hom}_{A}\left(B, J^{\bullet}\right)\right)\right) \\
& \simeq H^{i}\left(\operatorname{Hom}_{A}\left(B, \operatorname{Hom}_{A}\left(M, J^{\bullet}\right)\right)\right) \\
& \simeq \operatorname{Hom}_{A}\left(B, H^{i}\left(\operatorname{Hom}_{A}\left(M, J^{\bullet}\right)\right)\right)(B \text { is } A \text {-free }) \\
& =\operatorname{Hom}_{A}\left(B, \operatorname{Ext}_{A}^{i}(M, N)\right) .
\end{aligned}
$$

Now suppose for some fixed $i>0$ (therefore for all $i>0$ by the first part of the proof) that

$$
\operatorname{Ext}_{R}^{i}\left(f^{n} R, M\right)=0
$$

i.e.

This implies that

$$
\operatorname{Ext}_{R_{n}}^{i}\left(S / \mathbf{x}, \widetilde{F}_{S}^{n}(M)\right)=0
$$

$$
\operatorname{Hom}_{R_{n}}\left(\widehat{f}_{R_{n+1}}, \operatorname{Ext}_{R_{n}}^{i}\left(S / \mathbf{x}, \widetilde{F}_{S}^{n}(M)\right)\right)=0,
$$

where $\widehat{f}$ denotes $f_{S} \otimes_{S} R_{n}: R_{n} \rightarrow R_{n+1}$ (base change). Since ${ }^{\widehat{f}} R_{n+1}$ is free over $R_{n}$, by the Lemma, we have

$$
\operatorname{Ext}_{R_{n+1}}^{i}\left(\operatorname{Hom}_{R_{n}}\left(\widehat{f}_{R_{n+1}}, S / \mathbf{x}\right), \operatorname{Hom}_{R_{n}}\left(\widehat{f}_{R_{n+1}}, \widetilde{F}_{S}^{n}(M)\right)\right)=0,
$$

i.e.

$$
\operatorname{Ext}_{R_{n+1}}^{i}\left(\widetilde{F}_{S}(S / \mathbf{x}), \widetilde{F}_{S}^{n+1}(M)\right)=0 .
$$

We claim that, as $S / \mathbf{x}^{p}$-modules,

$$
\widetilde{F}_{S}(S / \mathbf{x}) \simeq S / \mathbf{x}^{p} .
$$

To see this, first note that by adjointness

$$
\begin{aligned}
\widetilde{F}_{S}(S / \mathbf{x}) & =\operatorname{Hom}_{S}\left({ }^{f} S, S / \mathbf{x}\right) \\
& \simeq \operatorname{Hom}_{S / \mathbf{x}}\left(S / \mathbf{x} \otimes_{S}{ }^{f} S, S / \mathbf{x}\right) \\
& =\operatorname{Hom}_{S / \mathbf{x}}\left({ }^{\widetilde{f}}\left(S / \mathbf{x}^{p}\right), S / \mathbf{x}\right),
\end{aligned}
$$

where $\tilde{f}$ is the map $\tilde{f}^{n}$ in the factorization (1) with $n=1$. Then observe that since $S / \mathbf{x}$ and $S / \mathbf{x}^{p}$ are complete intersections and $S / \mathbf{x}^{p}$ is a finitely generated $S / \mathbf{x}$ module via $\tilde{f}, \operatorname{Hom}_{S / \mathbf{x}}\left(\tilde{f}\left(S / \mathbf{x}^{p}\right), S / \mathbf{x}\right)$ is a canonical module for $S / \mathbf{x}^{p}$ and hence is isomorphic to $S / \mathbf{x}^{p}$ as an $S / \mathbf{x}^{p}$-module. 
It follows that $\widetilde{F}_{S}(S / \mathbf{x})$ is isomorphic to $S / \mathbf{x}^{p}$ as an $R_{n+1}$-module via the natural map $R_{n+1} \rightarrow R_{1}$ (i.e. the natural map $S / \mathbf{x}^{p^{n+1}} \rightarrow S / \mathbf{x}^{p}$ ).

Replacing $\widetilde{F}_{S}(S / \mathbf{x})$ in $(3)$ by $S / \mathbf{x}^{p}$, we obtain

$$
\operatorname{Ext}_{R_{n+1}}^{i}\left(S / \mathbf{x}^{p}, \widetilde{F}_{S}^{n+1}(M)\right)=0 .
$$

Consider the following short exact sequences:

$$
0 \rightarrow S /\left(x_{1}, x_{2}^{p}, \ldots, x_{r}^{p}\right) \stackrel{\lambda=x_{1}^{p}}{\longrightarrow} S /\left(x_{1}^{p+1}, x_{2}^{p}, \ldots, x_{r}^{p}\right) \rightarrow S / \mathbf{x}^{p} \rightarrow 0
$$

and

$$
0 \rightarrow S / \mathbf{x}^{p} \stackrel{x_{1}}{\rightarrow} S /\left(x_{1}^{p+1}, x_{2}^{p}, \ldots, x_{r}^{p}\right) \stackrel{\mu}{\rightarrow} S /\left(x_{1}, x_{2}^{p}, \ldots, x_{r}^{p}\right) \rightarrow 0 .
$$

Apply $\operatorname{Hom}_{R_{n+1}}\left(-, \widetilde{F}_{S}^{n+1}(M)\right)$ to $(5)$. From the associated long exact sequence and (4), we obtain an isomorphism (induced by $\lambda$ )

$$
\begin{aligned}
& \operatorname{Ext}_{R_{n+1}}^{i}\left(S /\left(x_{1}, x_{2}^{p}, \ldots, x_{r}^{p}\right), \widetilde{F}_{S}^{n+1}(M)\right) \\
\simeq & \operatorname{Ext}_{R_{n+1}}^{i}\left(S /\left(x_{1}^{p+1}, x_{2}^{p}, \ldots, x_{r}^{p}\right), \widetilde{F}_{S}^{n+1}(M)\right) .
\end{aligned}
$$

Similarly, applying $\operatorname{Hom}_{R_{n+1}}\left(-, \widetilde{F}_{S}^{n+1}(M)\right)$ to (6), we get a map (induced by $\mu$ )

$$
\begin{aligned}
& \operatorname{Ext}_{R_{n+1}}^{i}\left(S /\left(x_{1}, x_{2}^{p}, \ldots, x_{r}^{p}\right), \widetilde{F}_{S}^{n+1}(M)\right) \\
\rightarrow & \operatorname{Ext}_{R_{n+1}}^{i}\left(S /\left(x_{1}^{p+1}, x_{2}^{p}, \ldots, x_{r}^{p}\right), \widetilde{F}_{S}^{n+1}(M)\right),
\end{aligned}
$$

which is an isomorphism for $i \geq 2$ and a surjection for $i=1$.

The composition of maps $\lambda$ in (5) and $\mu$ in (6) is the following multiplication:

$$
S /\left(x_{1}, x_{2}^{p}, \ldots, x_{r}^{p}\right) \stackrel{x_{1}^{p}}{\longrightarrow} S /\left(x_{1}, x_{2}^{p}, \ldots, x_{r}^{p}\right),
$$

which is a 0-map. By (7) and (8), it induces

$$
\operatorname{Ext}_{R_{n+1}}^{i}\left(S /\left(x_{1}, x_{2}^{p}, \ldots, x_{r}^{p}\right), \widetilde{F}_{S}^{n+1}(M)\right) \stackrel{x_{1}^{p}}{\rightarrow} \operatorname{Ext}_{R_{n+1}}^{i}\left(S /\left(x_{1}, x_{2}^{p}, \ldots, x_{r}^{p}\right), \widetilde{F}_{S}^{n+1}(M)\right),
$$

which is an isomorphism for $i \geq 2$ and a surjection for $i=1$. Hence

$$
\operatorname{Ext}_{R_{n+1}}^{i}\left(S /\left(x_{1}, x_{2}^{p}, \ldots, x_{r}^{p}\right), \widetilde{F}_{S}^{n+1}(M)\right)=0 .
$$

Repeating this process $(r-1)$ times, we obtain

$$
\operatorname{Ext}_{R_{n+1}}^{i}\left(S / \mathbf{x}, \widetilde{F}_{S}^{n+1}(M)\right)=0,
$$

i.e.

$$
\operatorname{Ext}_{R}^{i}\left(f^{n+1} R, M\right)=0
$$

for all $i>0$.

Therefore by induction, $\operatorname{Ext}_{R}^{i}\left({ }^{f^{k}} R, M\right)=0$ for all $k \geq n$ and all $i>0$.

Finally, the assertion in the Theorem follows immediately from the following result due to Herzog ( $[\mathrm{H}]$, Theorem 5.2): If $M$ is a finitely generated module over $R$, then $M$ is of finite injective dimension if and only if $\operatorname{Ext}_{R}^{i}\left(f^{n} R, M\right)=0$ for all $i>0$ and infinitely many $n$.

Remark. The above theorem actually characterizes finitely generated modules with finite projective dimension also, since it is well known that over Gorenstein ring, a finitely generated module is of finite projective dimension if and only if it is of 
finite injective dimension. To sum up, if $M$ is a finitely generated module over a local complete intersection $R$, then the following are equivalent:

(i) $\operatorname{proj} \operatorname{dim}_{R} M<\infty$,

(ii) $\operatorname{Tor}_{i}^{R}\left(M, f^{n} R\right)=0$ for some fixed (all) $i, n>0$,

(iii) $\operatorname{Ext}_{R}^{i}\left(f^{n} R, M\right)=0$ for some fixed (all) $i, n>0$,

(iv) $\operatorname{inj} \operatorname{dim}_{R} M<\infty$.

\section{ACKNOWLEDGEMENTS}

I thank Professor Sankar Dutta for his suggestions and comments. I also thank the referee for suggestions improving the presentation of this note.

\section{REFERENCES}

[A-M] L. Avramov and C. Miller, Frobenius powers of complete intersections, Math. Research Letters, vol. 8, no. 1 \& 2, (2001), 225-232. MR.1825272 (2002b:13022)

[D] S.P. Dutta, On Modules of Finite Projective Dimension, Proc. Amer. Math. Soc. 131 (2003), no. 1, 113-116. MR.1929030 (2003j:13016)

$[\mathrm{H}] \quad$ J. Herzog, Ringe de Charakteristik p und Frobenius-Funktoren, Math Z. 140 (1974), 67-68. MR0352081 (50:4569)

[K] E. Kunz, Characterization of regular local rings for characteristic p, Amer. J. Math. 91 (1969), 772-784. MR0252389 (40:5609)

Department of Mathematics, University of Illinois at Urbana-Champaign, Urbana, ILLINOIS 61801

E-mail address: jinjiali@math.uiuc.edu 